\title{
On Potential Design Impacts of Electromigration Awareness
}

\author{
Andrew B. Kahng ${ }^{\dagger \dagger}$, Siddhartha Nath ${ }^{\dagger}$ and Tajana S. Rosing \\ ${ }^{\dagger}$ CSE and ${ }^{\ddagger}$ ECE Departments, UC San Diego, La Jolla, CA 92093 \\ \{abk, sinath, tajana $\} @$ ucsd.edu
}

\begin{abstract}
Reliability issues significantly limit performance improvements from Moore's-Law scaling. At $45 \mathrm{~nm}$ and below, electromigration (EM) is a serious reliability issue which affects global and local interconnects in a chip and limits performance scaling. Traditional IC implementation flows meet a 10-year lifetime requirement by overdesigning and sacrificing performance. At the same time, it is well-known among circuit designers that Black's Equation [2] suggests that lifetime can be traded for performance. In our work, we carefully study the impacts of EM-awareness on IC implementation outcomes, and show that circuit performance does not trade off so smoothly with mean time to failure (MTTF) as suggested by Black's Equation. We conduct two basic studies: EM lifetime versus performance with fixed resource budget, and EM lifetime versus resource with fixed performance. Using design examples implemented in two process nodes, we show that performance scaling achieved by reducing the EM lifetime requirement depends on the $E M$ slack in the circuit, which in turn depends on factors such as timing constraints, length of critical paths and the mix of cell sizes. Depending on these factors, the performance gain can range from $10 \%$ to $80 \%$ when the lifetime requirement is reduced from 10 years to one year. We show that at a fixed performance requirement, power and area resources are affected by the timing slack and can either decrease by $3 \%$ or increase by $7.8 \%$ when the MTTF requirement is reduced. We also study how conventional EM fixes using per net Non-Default Rule (NDR) routing, downsizing of drivers, and fanout reduction affect performance at reduced lifetime requirements. Our study indicates, e.g., that NDR routing can increase performance by up to $5 \%$ but at the cost of $2 \%$ increase in area at a reduced 7 -year lifetime requirement.
\end{abstract}

\section{INTRODUCTION}

At deep-submicron process nodes, electromigration (EM) poses significant reliability challenges which prevent further scaling of chip performance [18]. Electromigration causes shorts and voids in metal interconnects, leading to failures of the interconnects and decreased mean time to failure (MTTF) of the chip. ${ }^{1}$ Particularly for copper metallization at $45 \mathrm{~nm}$ and below, EM becomes a strong limiter of current density scaling [28]. Black's Equation [2] models median lifetime with respect to EM failures as

$$
t_{50}=\frac{A^{*}}{J^{n}} \cdot e^{\frac{E_{a}}{k T}}
$$

where $t_{50}$ is the median lifetime, $A^{*}$ is a geometry-dependent constant, $J$ is the current density, $n$ is a model parameter for current density, $E_{a}$ is the activation energy of metal ions, $T$ is the temperature of the interconnect, and $k$ is Boltzmann constant. The median lifetime $\left(t_{50}\right)$ is approximated as the MTTF of the interconnect. In our work, which focuses on (AC) signal EM, we use the root mean square (RMS) current density.

Besides the long-standing issue of DC electromigration, which affects power delivery networks, AC EM has become a serious concern for interconnects that carry clock and logic signals. AC EM limits become tighter, and EM design violations worsen, with technology scaling since (1) on-current of drivers increases with smaller channel lengths, (2) widths of interconnects decrease, (3) faster switching of transistors increases frequency of operation, and (4) capacitive loads increase due to scaling non-idealities. Together, these factors cause a significant increase in current density, leading to vulnerabilities in the design. Figures 1(a) and (b) show the exponential increase of current density, along with the exponential decrease of wire width, with successive technology nodes.

Figure INTC 9 from the Interconnect Chapter of the 2011 International Technology Roadmap for Semiconductors (ITRS) [31] indicates that maximum current density limits (from EM reliability considerations) become the barrier to further frequency scaling from 2018

\footnotetext{
${ }^{1}$ In the constant failure rate region of the reliability bathtub curve, reliability is modeled using the exponential distribution. In the exponential distribution, the median is $\ln (2)$ times the mean. Our use of the MTTF term throughout this paper refers to the mean measure; however, using the median measure will not change the trends in our results.
}

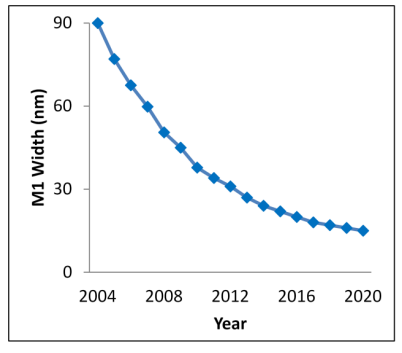

(a)

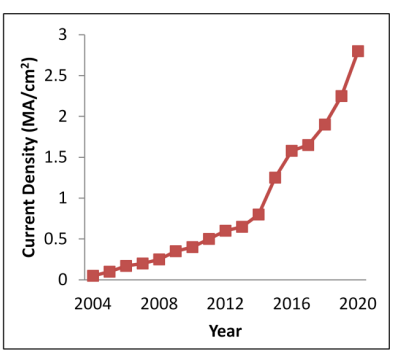

(b)
Fig. 1. (a) Wire width scaling, and (b) current density scaling [28], [31].

onwards. This motivates a closer look at the potential benefits of improved analysis, and/or relaxation, of EM reliability limits. Today, most circuits are still designed to meet 10 -year lifetime requirements [13]; however, recent trends suggest that some types of high-volume products are replaced by users after much smaller lifetimes. For example, in the United States people replace their cell phones roughly every two years [34] and laptops every three to five years [35]. Servers are replaced by enterprises every three to seven years [36]. From Black's Equation (1), MTTF can be traded for an increase in the RMS current, $I_{r m s}$, which can be further used to increase the maximum operating frequency $\left(F_{\max }\right)$ of a design. Therefore, one may seek to design products with reduced MTTF requirements and use the resulting EM slack (see discussion in Section III) to improve $F_{\max }$. (Alternatively, EM slack may be used to save chip area and power resources while keeping the same product performance.)

However, the MTTF vs. performance relation is not as straightforward as suggested by Black's Equation. Different designs have different performance scaling for the same MTTF tradeoff, and the scaling depends on several other factors such as EM slack, upper bounds on area and peak temperature. In our work, we perform two basic studies. Experiment 1: We study MTTF vs. $F_{\max }$ tradeoffs at fixed budgets for area, power and temperature.

Experiment 2: We study MTTF vs. area and power tradeoffs at a fixed performance requirement.

Experiment 1 leads to three key observations. (1) We show that $F_{\text {max }}$ scaling across different circuits is dependent on EM slack which is determined by timing constraints, mixes of cell sizes, and lengths of critical paths in the circuit. (2) We identify the dominating constraints (area, EM or temperature) that can limit frequency scaling as lifetime requirements range from 10 years to one year. (3) We empirically determine the design guideline that area and temperature limit $F_{\max }$ scaling only when the lifetime requirement is less than four years. Experiment 2 leads to two key observations. (4) Where timing constraints are loose, resource usage can reduce as MTTF reduces; on the other hand, (5) where timing constraints are tight, resource usage will increase as MTTF reduces (hence, EM-awareness will give no benefit). We also compare how conventional fixes for EM and signal integrity (SI) issues affect performance. Our results show, e.g., that up to a lifetime of seven years, Non-Default Rules (NDRs) created by comprehending $I_{r m s}$ violations on each net are effective in improving performance by up to $5 \%$ at an area cost of $2 \%$. Further, downsizing drivers and reducing fanouts are less effective levers to improve performance (the ratio of increase in performance to increase in area is 1.5 whereas it is 2.5 for NDR routing).

Our main contributions are as follows.

1) Our study shows that EM slack is needed to maximize $F_{\max }$ when lifetime requirement is reduced. In general, 20\% EM slack allows increase of $F_{\max }$ by $20 \%$ and $83 \%$ EM slack allows increase of $F_{\max }$ by $80 \%$ when the lifetime requirement is reduced to one year.

2) Our studies of MTTF vs. area and power at a fixed $F_{\max }$ show 
that tightness vs. looseness of timing constraints determine area and power trends at reduced MTTF.

3) Our results give front-end designers and architects insight into lifetime tradeoffs, particularly opportunities to scale $F_{\max }$ at no area cost. Physical design engineers can obtain new understanding of performance vs. area tradeoffs at different lifetimes, when NDR routing, driver downsizing and fanout reductions are the available levers for EM fixes.

The rest of our paper is organized as follows. Section II discusses related work, and Section III gives added background on electromigration and implications of Black's Equation. Section IV describes our analytical model, problem formulation and description of our optimization flow. In Section V we discuss validation and results of our optimization flow. The paper concludes with directions for future work in Section VI.

\section{RELATED WORK}

Previous works on electromigration can be broadly classified as (1) models to accurately estimate EM MTTF, (2) impact of technology scaling and architectural techniques to mitigate EM failures, and (3) synthesis and physical design techniques to reduce current density violations and meet lifetime of the chip.

The EM modeling literature begins with Black's proposal [2] of an analytical model for EM median time to failure $\left(t_{50}\right)$. Liew et al. [9] extend Black's Equation to propose DC and AC lifetime models by accounting for EM healing during vacancy relaxation time. Clement [3] uses analytical models for EM failure to describe situations when void growth or stress build-up are the dominant factors. Lloyd [10] proposes void growth and nucleation models to explain that EM failure is also governed by the time it takes for a void to grow to its failure point. These models are essentially parametric modifications of Black's Equation. Orio et al. [5] study the well-known EM failure models to identify gaps in models and implementation. Shatzkes et al. [15] empirically demonstrate that the current density exponent in Black's Equation should be $n=2$. Wu et al. [19] and Lu et al. [12] propose EM lifetime models that comprehend joule heating and thermal gradient effects on interconnects.

In the architecture literature, Srinivasan et al. [17] consider requirements to change conventional microprocessor architectures such that they comprehend reliability issues of the underlying hardware. Romanescu et al. [14] propose core cannibalization to improve lifetime performance by sharing resources between cores when there are hard faults. Their architecture brings implementation challenges due to significant changes in hardware design (e.g., multiplexing of every resource in every pipeline stage). Lu et al. [11] propose to improve performance - in the sense of reclaiming excess design margin - by accounting for temperature gradients in reliability models.

Synthesis and physical design mechanisms include work by Dasgupta et al. [4], who propose a synthesis methodology for highreliability netlists by taking into account EM degradation arising from switching activity correlations on a microprocessor data transfer bus. Jerke et al. [7] propose a design rule methodology to check current density violations. They use a quasi-3D model to hierarchically calculate current density and perform thermal simulations to check violations in wires and vias. Lienig et al. [8] use post-route area adjustment of critical net structures to size wires and vias and thereby fix current density violations. Banerjee et al. [1] study the extent of EM failure under AC bipolar stress and joule heating in signal nets. Hunter [6] demonstrates that joule heating will limit the peak current densities in circuits and will pose a bigger reliability issue on top of EM failures due to current density violations. Finally, recent whitepapers from Synopsys [26] and Cadence [21], [22] propose ways to fix EM and other signal integrity violations after routing to meet lifetime requirements, but do not quantify impacts on design metrics such as area, congestion and power.

In all of these previous works on EM, the goal has been to meet lifetime bounds by accurately estimating the degradation, then applying fixes using physical design techniques. Our work takes a very different perspective: we directly examine the potential design impacts of trading off EM lifetime bounds, whether to increase the performance of the design, or to reduce area and power given fixed performance and thermal budget.

\section{IMPLICATIONS OF BLACK'S EQUATION}

We now give a brief background on EM, the design and runtime parameters that affect EM, and implications of Black's Equation. As noted above, EM causes voids and shorts in IC interconnects through momentum transfer between metal ions and conducting electrons; this reduces the reliability of IC interconnects. EM lifetime is affected by design parameters such as wire width, fanout, driver size, and operating voltage - since all of these affect current density. Runtime parameters that affect EM are switching activity $(\alpha)$, frequency, and temperature. Figure 2 shows the relation to EM MTTF of these factors, with positive (negative) correlations to MTTF shown as red (blue) directed edges.

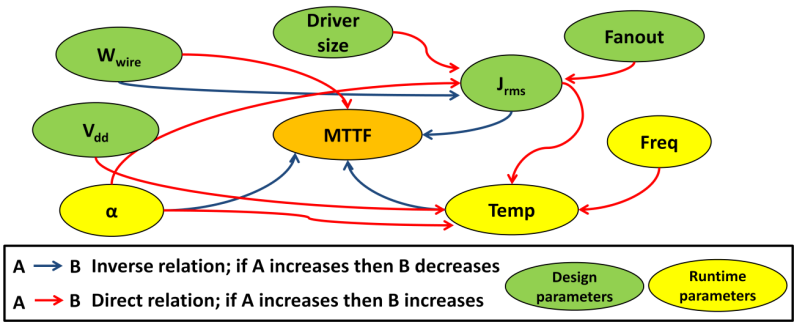

Fig. 2. Design and runtime factors affecting EM MTTF.

EM slack is a function of the RMS current $\left(I_{r m s-n e t}\right)$ and RMS current limit $\left(I_{r m s-l i m i t}\right)$ according to the technology Library Exchange Format (LEF) file [32]. Under AC stress the EM slack is given by

$$
I_{r m s-n e t}=I_{r m s-\text { limit }} \cdot \sqrt{\frac{M T T F_{d e f}}{M T T F_{r e d}}}
$$

where $M T T F_{d e f}$ is the default lifetime requirement (typically 10 years) and $M T T F_{\text {red }}$ is the reduced lifetime requirement for the design. EMawareness can improve performance if there is positive EM slack, i.e., $I_{r m s-n e t}-I_{r m s-l i m i t}>0$. Therefore, for any circuit, Equation (2) determines the theoretically available performance improvement when MTTF is reduced. From Black's Equation, $I_{r m s-n e t}$ is given by

$$
\begin{aligned}
I_{r m s-n e t} & =\left(\frac{1}{M T T F} \cdot A^{*}(W \cdot H)^{n} e^{\frac{E a}{k T}}\right)^{\frac{1}{n}}=K_{1} \cdot\left(\frac{1}{M T T F}\right)^{\frac{1}{n}} \\
K_{1} & =\left(A^{*}(W \cdot H)^{n} e^{\frac{E a}{k T}}\right)^{\frac{1}{n}}
\end{aligned}
$$

where $W$ and $H$ respectively denote the width and height of a wire segment of a net. With all other parameters remaining constant, Equation (3) shows that $I_{r m s-n e t}$ scales inversely with MTTF.

In an actual circuit, $I_{r m s-n e t}$ of a net is given by

$$
I_{r m s-n e t}=C \cdot V_{d d} \cdot \sqrt{\alpha \cdot F_{\max }\left(\frac{1}{t_{r}}+\frac{1}{t_{f}}\right)}
$$

where $C$ is the sum of load and wire capacitances, $V_{d d}$ is the operating voltage, $t_{r}$ and $t_{f}$ are respectively the rise and fall times of the driver, and $\alpha$ is the switching activity on the net with $0 \leq \alpha \leq 1$.

By combining Equations (5) and (3), we may express $F_{\max }$ as

$$
\begin{aligned}
F_{\max } & =\left(\frac{K_{1}}{K_{2}}\right)^{2} \cdot \frac{1}{C^{2} V_{d d}^{2}} \cdot \frac{1}{\alpha} \cdot\left(\frac{1}{M T T F}\right)^{\frac{2}{n}} \\
K_{2} & =\sqrt{\frac{1}{t_{r}}+\frac{1}{t_{f}}}
\end{aligned}
$$

Notice that Equation (6) is quite useful: it shows how performance and reliability interact when the synthesis, place and route (SP\&R) degrees of freedom are only gate sizing (affects $K_{2}$ and $C$ ) and wire width/spacing sizing (affects $K_{1}$ and $C$ ). Conventional methods for EM fixes use these degrees of freedom. In our work, we use these degrees of freedom along with EM slack as given by Equation (2) to study potential circuit performance at reduced MTTF requirements.

\section{DESIGN OF EXPERIMENTS}

We now describe our experimental flows. Experiment 1 requires us to find the highest possible maximum frequency of a design, subject to upper bounds on area, peak temperature and number of critical EM 
violations, for a given reduced MTTF requirement. Formally, we maximize $F_{\max }$

subject to

$$
\begin{aligned}
& T_{\text {peak }} \leq T_{U B} \\
& U I L_{\text {eff }} \leq U T I L_{U B} \\
& \sum\left(\frac{I_{r m s-n e t}}{I_{\text {rms-limit }}}>1.1\right) \leq \text { VIONETS } S_{U B}
\end{aligned}
$$

where $F_{\max }$ is the maximum operating frequency (which we want to maximize), $T_{\text {peak }}$ is the peak temperature of the design, $T_{U B}$ is the upper bound on the peak temperature of the design, $U T I L_{e f f}$ is the post-P\&R effective utilization of the design, $U T I L_{U B}$ is the upper bound on the effective utilization (which is the upper bound on the area), and VIONETS $S_{U B}$ is the maximum number of wire segments that violate $I_{r m s-l i m i t}$ by more than $10 \%$ (= our upper bound on critical EM violations).

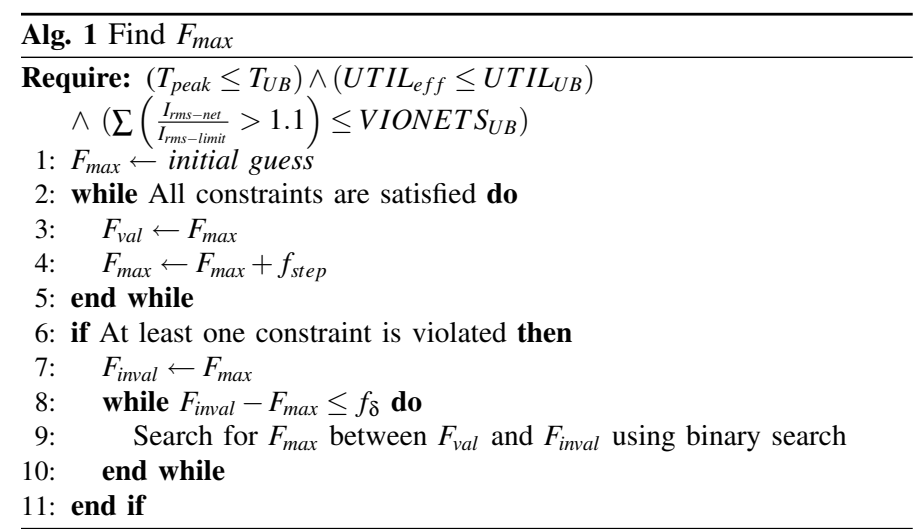

We perform this optimization using Algorithm 1, which seeks a (global optimum) maximum value for $F_{\max }$. We begin with an initial guess for $F_{\max }$ and greedily increment this by $f_{\text {step }}$ as long as all constraints are satisfied. The last valid frequency that satisfies all the constraints is stored in $F_{v a l}$. If a violation occurs, the violating frequency is stored in $F_{\text {inval }}$. As long as $F_{\text {inval }}-F_{\text {max }} \leq f_{\delta},{ }^{2}$ binary search is used to find the $F_{\max }$ which is bounded by $F_{\text {inval }}$ and $F_{v a l}$. The greatest frequency that meets all the constraints within this bound is the $F_{\max }$, since frequency keeps increasing by $f_{\text {step }}$ in each loop until a violation is met. When a violation occurs, binary search efficiently determines the final $F_{\max }$. The final $F_{\max }$ differs from the frequency that violates at least one constraint by $f_{\delta}$ and hence is the maximum achievable $F_{\max }$ for the design. We use Algorithm 1 in the flow described in Section IV-B below.

Experiment 2 requires us to fix timing constraints for a circuit, sweep MTTF from 10 years down to one year, and observe changes in area and power. We keep designs free from EM violations by construction, by limiting cell sizes as shown in Table II (see discussion in Section V).

\section{A. LEF Current Density Characterization}

In both experiments, we characterize the current density limits for each metal layer in the technology LEF [32] for a given reduced MTTF requirement $\left(M T T F_{\text {red }}\right)$, switching activity at the primary inputs (PIs) $\left(\alpha_{P I}\right)$, and peak temperature $\left(T_{U B}\right)$. We assume that at $T_{U B}$, the design is free from electromechanical stress and that $T_{U B}$ includes joule heating effect due to AC stress on the signal interconnects. The new peak and RMS current density limits of each metal layer in the LEF, $J_{\text {peak-new }}$ and $J_{r m s-n e w}$ respectively, are calculated from the default $J_{\text {peak-def }}$ and $J_{r m s-d e f}$ using Black's Equation:

$$
\begin{gathered}
J_{\text {peak-new }}=\left(\frac{M T T F_{\text {def }}}{M T T F_{\text {red }}} \cdot J_{\text {peak }- \text { def }}^{n} \cdot e^{\frac{E_{a}}{k}\left[\frac{1}{T_{U B}}-\frac{1}{T_{\text {def }}}\right]}\right)^{\frac{1}{n}} \\
J_{\text {rms-new }}=\left(\frac{M T T F_{\text {def }}}{M T T F_{\text {red }}} \cdot J_{\text {rms }- \text { def }}^{n} \cdot\left(\frac{\alpha_{P I}}{\alpha_{\text {def }}}\right)^{\frac{n}{2}} \cdot e^{\frac{E_{a}}{k}\left[\frac{1}{T_{U B}}-\frac{1}{T_{\text {def }}}\right]}\right)^{\frac{1}{n}}
\end{gathered}
$$

where $M T T F_{d e f}$ is the default lifetime defined by the foundry, $\alpha_{d e f}$ is the default switching activity of metal layer, $T_{d e f}$ is the default

\footnotetext{
${ }^{2} f_{\delta}$ is the minimum tolerable frequency difference with $F_{\text {inval }}$.
}

temperature at which the LEF is characterized by the foundry, $n$ is the model parameter for current density, $E_{a}$ is the activation energy of the interconnect metal or metal alloy, and $k$ is Boltzmann constant.

\section{B. $F_{\max }$ Flow}

Figure 3 shows our flow for Experiment 1. The flow is described in detail by the following steps.

1) Start with an initial timing constraint (SDC [30] file).

2) Synthesize a design from its RTL description, cell library with cell mixes for different lifetime requirements and SDC.

3) Characterize current density limits in each metal layer in the technology LEFs based on $T_{U B}$, switching activity, and reduced MTTF requirements $\left(M T T F_{\text {red }}\right)$ using Black's Equation as described in Section IV-A.

4) Place and route the post-synthesis netlist using the newly characterized technology LEF from the previous step.

5) Perform post-route extraction, timing and signal integrity (SI) analysis. Fix EM violations using P\&R tool commands.

6) Calculate peak temperature of the design $\left(T_{\text {peak }}\right)$ from chip and core areas, ambient temperature $\left(T_{a m b}\right)$, and power using Hotspot [16] calibrated to a $45 \mathrm{~nm}$ Qualcomm SoC package.

7) Check that the number of wire segments violating the $I_{r m s}$ limit by more than $10 \%$ is less than VIONET $S_{U B}$, slack is met, $T_{\text {peak }} \leq T_{U B}$ and $U T I L_{e f f} \leq U T I L_{U B}$.

8) If all constraints are met, then increase frequency (decrease clock period in SDC) using binary search. If any constraint is violated, decrease frequency (increase clock period) using binary search.

9) If the next frequency has already given a valid solution, then exit, else repeat the flow from Step 2 using a frequency obtained from the previous step.

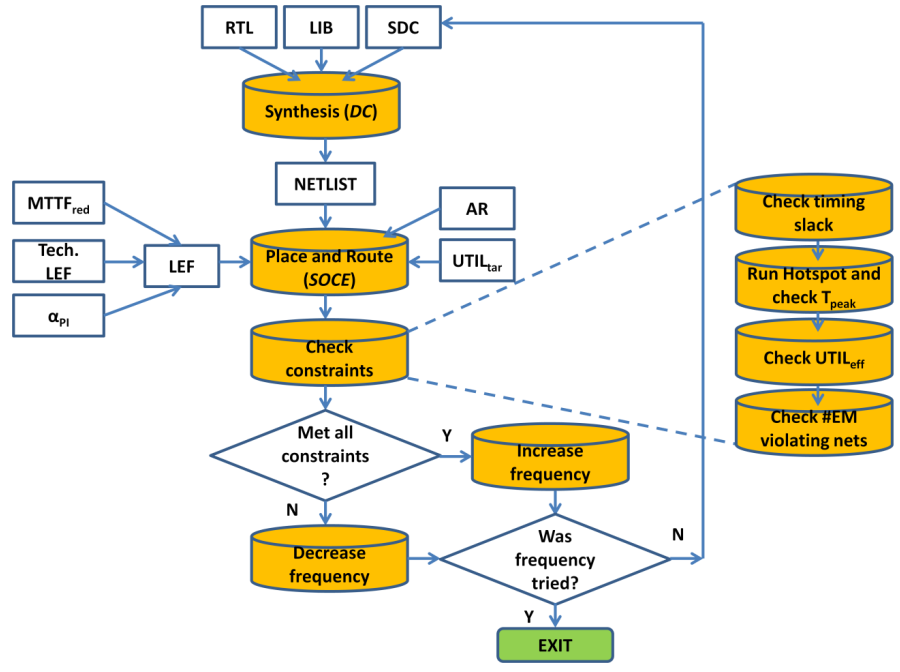

Fig. 3. $F_{\max }$ determination flow.

\section{Post-Route EM Fix Flow}

In both of our experiments, we fix critical EM violations after routing to meet required lifetimes. We use a conventional physical design (PD) methodology that relies on NDRs in engineering change order (ECO) routing to widen wires and spacings, along with fanout reduction and downsizing of drivers [26], [21], [7]. We also study how such standard methods can affect performance and area of a design. We implement these techniques using Cadence SOC Encounter vEDIIO.1 (SOCE) [25], [20]. Figure 4 shows our flow to create NDRs per net; the following steps describe implementation in SOCE.

1) Group EM critical nets by $I_{r m s}$ (which is a function of the switching activity on the net) and create NDRs for wire-width and spacing depending on the extent of $I_{r m s}$ violation. ${ }^{3}$

2) Execute command verifyACLimit in SOCE to find the extent of current density violations vis-a-vis LEF in each metal layer, as described in Section IV-A. Apply NDRs to grouped nets

${ }^{3}$ To avoid creating too many NDRs, nets with $I_{r m s}$ violation in the range $+10 \%$ to $-10 \%$ are grouped together. 
using the command setAttribute -nets netname -non_default_rule rulename. If any of these nets are "FIXED" (such as clock nets), then convert them to SPECIAL nets for re-routing.

3) Select all violating nets for ECO routing using the command selectNet netname.

4) Perform ECO routing of these nets using the commands setNanoRouterMode -routeWithECO true; setNanoRouterMode -routeSelectedNetsOnly true; setNanoRouterMode

-routeStrictlyHonorNonDefaultRule true; globalDetailRoute. Then, verify AC limit violations after re-route. If there are $\mathrm{AC}$ limit violations, decrease fanout of these nets using the command set_max_fanout fanout and perform ECO routing.

5) If violations remain after the second ECO route, swap large drivers with smaller drivers and redo timing analysis. If no timing violations, then accept this new frequency and exit, else reduce frequency and run the flow from Section IV-B. If frequency $\leq F_{\text {max }}$ obtained from the flow in Section IV-B, then exit.

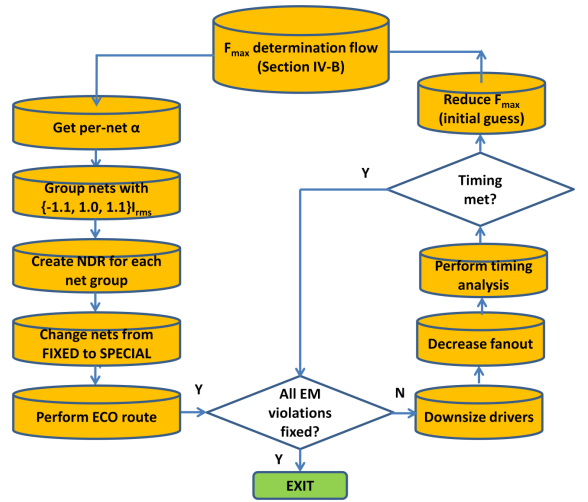

Fig. 4. Per-net NDRs flow to fix EM $I_{r m s}$ violations.

\section{VALIDATION AND RESULTS}

Table I describes the implementation parameters used in our experiments. We synthesize the design using Synopsys Design Compiler vD2010.03-SP1-64 [29] to obtain a post-synthesis netlist. We set floorplan aspect ratio to 1.0 and target utilization to $60 \%$, then place and route the post-synthesis netlist using Cadence SOC Encounter vEDI10.1 [25]. We reject any solution that has an effective utilization of more than $66 \%$ because we want to ensure that there is enough whitespace to fix any remaining EM violations by widening wires and re-routing the design using NDRs. To eliminate tool noise, we execute each SP\&R run five times by perturbing the timing constraints by $\pm 0.5 \%$ and $\pm 1 \%$. Our fully automated flow is implemented in bash and TCL scripts. The default MTTF for our study is 10 years [13], and the reduced MTTFs from nine to one years. Although we use three different switching activity factors, $(\alpha=0.05,0.2,0.5)$ in our experiments, we report results for $\alpha=0.05$ since this is a typical switching activity at primary inputs for practical workloads. We use six designs from [33] (JPEG encoder, AES cipher, Wishbone DMA controller, PCI bridge, $5 \times 5$ network-on-chip router and OpenSparcT1 fpu) and two standardcell libraries, TSMC45GS and TSMC65GPLUS. We present results of JPEG, AES and DMA because they are representative of all our observations.

As we alluded above, a standard "correct by construction" EM reliability methodology used by industry PD teams is to determine allowed cell strengths for SP\&R based on the EM lifetime requirement. Because EM fixes post-P\&R - e.g., ECO routing with new NDRs per violating net - are time-consuming and disruptive, the typical methodology goal is to choose cell strengths that will lead to at most several hundred EM violations at the conclusion of the automated SP\&R flow. We have performed analyses of our $65 \mathrm{~nm}$ and $45 \mathrm{~nm}$ cell libraries to determine cell strengths that result in at most $25 \mathrm{EM}$ violations in automated block implementation. Our flows apply the results of this analysis, which are shown in Table II.

\section{A. Experiment 1: MTTF vs. $F_{\max }$}

We validate the Section IV-B flow against the analytical model for EM slack in Equation (2) using three designs - JPEG, AES and DMA. Figures 5(a) and (b) respectively show the percentage of increase in
TABLE I

IMPLEMENTATION PARAMETERS

\begin{tabular}{|c|c|c|}
\hline Parameter & Definition & Value \\
\hline$T_{U B}$ & Peak temperature upper bound & $105^{\circ} \mathrm{C}$ \\
\hline$U T I L_{U B}$ & Effective utilization upper bound & $66 \%$ \\
\hline VIONETS $_{U B}$ & \# of EM violating net segments & 25 \\
\hline$M T T F_{\text {def }}$ & Default MTTF & 10 years \\
\hline$M T T F_{\text {red }}$ & Reduced MTTF & $\{9,8,7, \ldots, 2,1\}$ years \\
\hline$\alpha_{P I}$ & Switching activity at PIs & $\{0.05,0.2,0.5\}$ \\
\hline$T_{\text {amb }}$ & Ambient temperature & $45^{\circ} \mathrm{C}$ \\
\hline$T_{\text {def }}$ & Default temperature of LEF & $85^{\circ} \mathrm{C}$ \\
\hline$E_{a}$ & Activation energy of interconnect & $0.7 \mathrm{eV}$ \\
\hline$k$ & Boltzmann constant & $8.617 \times 10^{-5} \mathrm{eV} / \mathrm{K}$ \\
\hline$n$ & Current density model parameter & 2 \\
\hline$f_{\text {step }}$ & Frequency change step & $200 \mathrm{MHz}$ \\
\hline$f_{\delta}$ & Minimum tolerable frequency delta & $25 \mathrm{MHz}$ \\
\hline$U T I L_{\text {tar }}$ & Target utilization & $60 \%$ \\
\hline$A R$ & Floorplan aspect ratio & 1.0 \\
\hline
\end{tabular}

TABLE II

CELL STRENGTHS REQUIRED TO LIMIT THE NUMBER OF EM VIOLATIONS TO LESS THAN 25 AFTER SP\&R.

\begin{tabular}{|c|c|}
\hline MTTF (years) & Cell Strength \\
\hline$\{10,9,8\}$ & all cells up to X12 \\
\hline$\{7,6,5\}$ & all cells up to X16 \\
\hline$\{4,3\}$ & all cells up to X24 \\
\hline$\{2,1\}$ & all cells \\
\hline
\end{tabular}

the maximum frequency of these designs at $45 \mathrm{~nm}$ and $65 \mathrm{~nm}$, with $\alpha=0.05$. We observe that, unlike what is suggested by Black's Equation, frequency does not increase continuously for all types of circuits at both process nodes. As expected, frequency scaling is greater at $45 \mathrm{~nm}$ because EM slack is larger, cell areas are smaller and transistors are faster than at $65 \mathrm{~nm}$. Since $45 \mathrm{~nm}$ and $65 \mathrm{~nm}$ trends are similar, we use the $45 \mathrm{~nm}$ results as the backdrop for our observations. Figures 6(a) and (b) show the EM slack and timing slacks available in the circuits at each lifetime requirement; the positive slack indicates that none of the circuits is limited by tight timing constraints and that timing margin is available for further frequency scaling. Lifetime reduction can increase $F_{\max }$ proportional to the amount of EM slack available in the critical paths of the circuits. The key observations from this experiment are:

Observation 1: Positive EM slack, given by the extent of $I_{r m s}$ violation at each lifetime, determines the potential frequency scaling benefit of reduced MTTF. The JPEG design has frequency scaling benefit from reduced MTTF all the way to one year lifetime because $I_{r m s}$ violations offer sufficient EM slack that can be used to scale frequency. For example, at a two-year lifetime the violations are over $2 \mathrm{x}$ in the clock nets, so frequency increases by $18 \%$ when MTTF is reduced to one year. Figure 7(a) shows the percentage of EM violations remaining in the critical paths. For the constraints in our experiment, all violations are resolved at one-year lifetime. For the DMA design, $F_{\text {max }}$ increases by $60 \%$ when MTTF reduces from 10 to four years. Below four years, the violations are less than $20 \%$ and the flow cannot make use of the theoretical EM slack of $41 \% 4$ from Equation (2).

Observation 2: The EM slack in each circuit is determined by the mix of cells. According to Table II, EM slack increases as MTTF reduces because we increase the size of cells. Figures 8(a) and (b) show that cell size and frequency determine the total power. Large cells are more frequently used as MTTF reduces, which makes the ratio of leakage to total power nearly constant. EM slack is also a function of length of critical paths. At MTTF less than six years, JPEG and DMA have short critical paths (20 stages on average), so the switching activity on each net more closely tracks that of the PIs. This leads to more $I_{r m s}$ violations, which can be used as EM slack. AES, on the other hand, has longer critical paths (30 stages on average) below seven years MTTF, which makes switching activity on nets and wire capacitances (as well as net lengths) small; thus, the extent of $I_{r m s}$ violations is small. We see that AES has at most $27 \%$ performance increase despite having slack in timing and utilization as shown in Figures 6(b) and 7(b) respectively. Observation 3: For MTTF requirements below three years, area plays an important role in $F_{\max }$ scaling. Figure $7(\mathrm{~b})$ shows that the DMA design cannot scale frequency further when MTTF drops below seven years, because area upper bounds are violated, i.e., total cell area

\footnotetext{
${ }^{4}$ In Figure 6(a), EM slack is expressed as a percentage of $I_{r m s-l i m i t}$.
} 
exceeds available layout area. However, until MTTF is decreased to four years, these designs can increase frequency by different amounts at no area cost: frequency increases for AES, JPEG and DMA are $20 \%, 70 \%$ and $50 \%$, respectively. The potential for increased frequency without area cost may be motivation for front-end designers and architects to incorporate EM-awareness in their designs. Figures 8(a) and (b) respectively show that the percentage increase in total power grows as $F_{\max }$ increases, and that leakage power remains almost constant relative to total power.

Figures 9(a) and (b) show the dominating constraints for $F_{\max }$ scaling at different lifetimes for the $45 \mathrm{~nm}$ JPEG and DMA designs. For both designs, EM is the dominating constraint until a five-year MTTF. At one-year lifetime, area constraints prevent further $F_{\max }$ scaling. Figures 9(c) and (d) show the dominating constraints for these designs at $65 \mathrm{~nm}$. Again, EM is a dominant constraint until a six-year MTTF, but for lifetimes less than four years peak temperature becomes a dominating constraint as larger cells dissipate more power, and more instances are used to meet $F_{\max }$ constraints at $65 \mathrm{~nm}$.

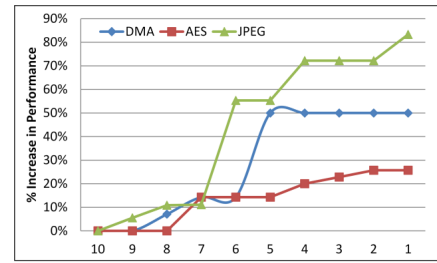

(a)

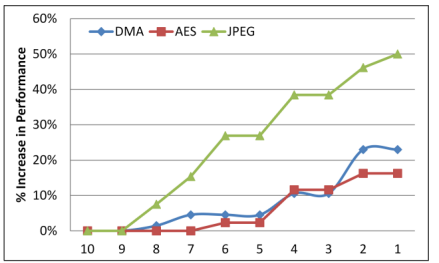

(b)
Fig. 5. Percentage increase in $F_{\max }$ at (a) $45 \mathrm{~nm}$ and (b) $65 \mathrm{~nm}$ due to reduced MTTF requirement.

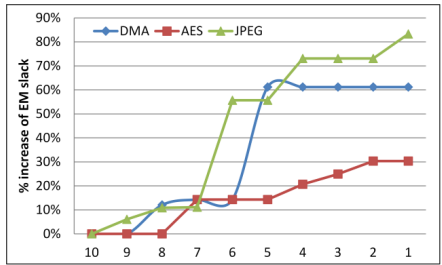

(a)

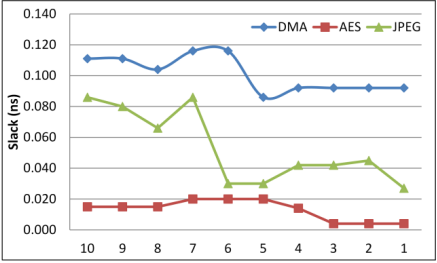

(b)
Fig. 6. (a) Percentage increase of EM slack and (b) timing slack in circuits at $45 \mathrm{~nm}$ due to reduced MTTF requirement.

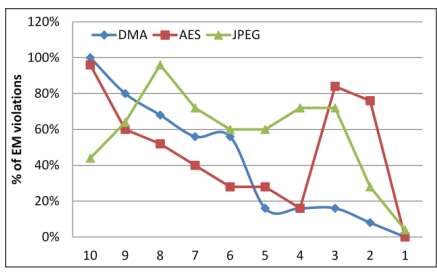

(a)

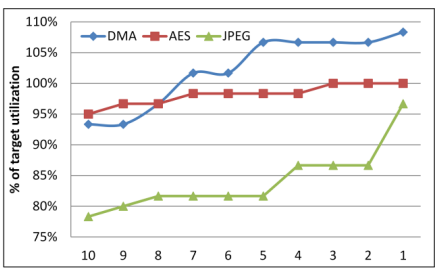

(b)
Fig. 7. (a) Percentage of critical paths with EM violations and (b) total cell area relative to available area at $45 \mathrm{~nm}$ as MTTF requirement is reduced.

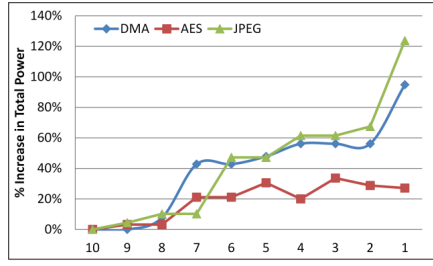

(a)

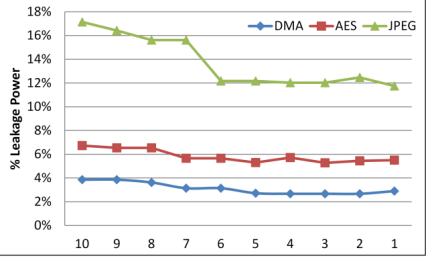

(b)
Fig. 8. Percentage of (a) increase in total power and (b) leakage power relative to total power as MTTF requirement is reduced.

\section{B. Experiment 2: MTTF vs. Area and Power}

Our Experiment 2 results show how timing constraints determine available scaling of area and power. We implement AES $(1100 \mathrm{MHz})$, JPEG $(850 \mathrm{MHz})$ and DMA $(2000 \mathrm{MHz})$ designs at $45 \mathrm{~nm}$ using the cell strengths at each MTTF in Table II. The constraints for JPEG are

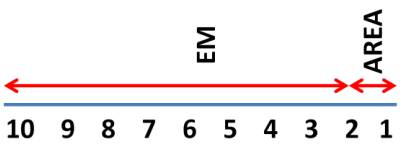

(a)

(c)

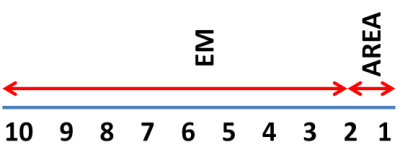

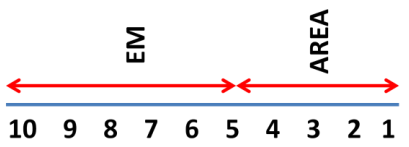

(b)

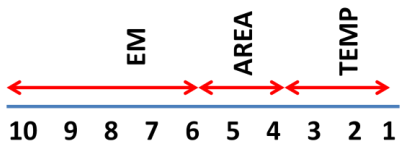

(d)
Fig. 9. Dominating constraints on $F_{\max }$ scaling at different MTTF limits: (a) JPEG 45nm, (b) DMA 45nm, (c) JPEG 65nm, (d) DMA 65nm.

loose, with 93ps of slack after SP\&R; DMA and AES have 2ps and $1.6 \mathrm{ps}$ of post-P\&R slack, respectively.

Observation 4: If a design has large positive timing slack at 10 years, then area and power decrease when MTTF is reduced. Figures 10(a) and (b) show the area and power reduction for JPEG. The positive slack at 10-year lifetime allows the design to replace small-sized buffers and inverters with large drivers when MTTF is reduced, thereby reducing the number of instances. The number of instances is also reduced with MTTF because EM violations are reduced, and post-route fixes do not need to add extra buffers when reducing fanouts or downsizing drivers. Collectively, these factors reduce area by $3.7 \%$ and power by $2.5 \%$.

Observation 5: If a design has little or no positive timing slack at 10year lifetime, then area and power increase when MTTF is reduced. For AES (and DMA), the constraint is very tight at 10 years. As MTTF reduces, smaller drivers are replaced by larger drivers to meet timing, but the number of instances does not decrease. Although the number of EM violations reduces with MTTF which, in turn, reduces the resources needed to fix these violations after routing, the number of instances does not reduce significantly. As MTTF reduces, these factors increase area by $2.02 \%$ and power by $7.08 \%$ as shown in Figures 11 (a) and (b). Hence, EM-awareness does not benefit circuits with tight timing constraints.

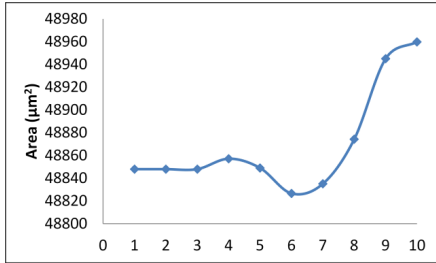

(a)

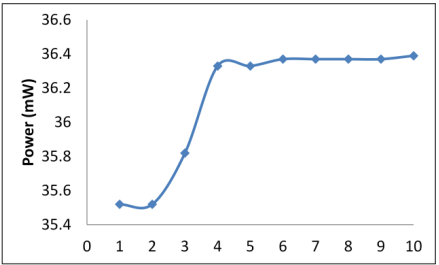

(b)
Fig. 10. JPEG (a) area and (b) power at $45 \mathrm{~nm}$.

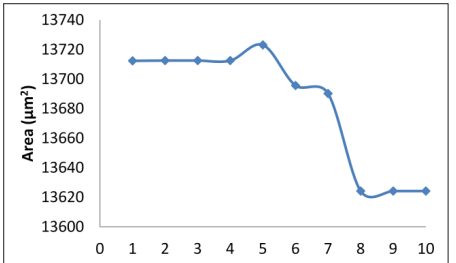

(a)

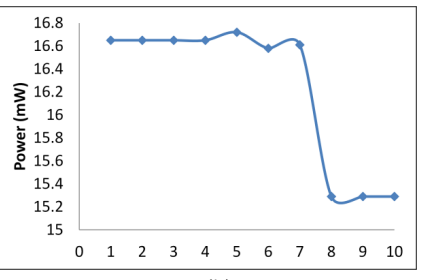

(b)
Fig. 11. AES (a) area and (b) power at $45 \mathrm{~nm}$.

\section{Performance and Area Impact of Conventional EM-Fix Methods}

We additionally study how conventional EM and SI fixing methods affect performance and area at reduced lifetime requirements. We sweep MTTF from 10 years down to one year and apply per-net NDRs, driver downsizing and fanout reduction fixes as described in Section IV-C to the three $45 \mathrm{~nm}$ testcases. Our analyses show that NDRs are more flexible in repairing EM violations.

Observation 6: Using NDRs to fix EM violations helps to increase $F_{\max }$ only until MTTF reaches seven years. Figure 12 shows that the increase in $F_{\max }$ is less than 5\% for all the designs. For JPEG, there is no increase although area increases by $2 \%$; this is because the extensive $I_{r m s}$ violations cannot be repaired by the conventional fixing methods. AES, however, gains about $4.65 \%$ in frequency by paying $0.4 \%$ area, since its smaller EM slack permits repair using conventional methods. 
Observation 7: When drivers are downsized, numbers of buffers and inverters increase to meet timing; when fanout is reduced, (too many) cloned buffers are added to meet clock skew. Figures 13(a) and (b) show how area and performance change by changing fanout and driver sizes, respectively, at MTTF of seven years for the AES design. A performance increase of $3 \%$ comes at the cost of a $1.86 \%$ increase in area. Compared to downsizing and fanout reduction, NDRs appear to improve $F_{\max }$ with less increase in area.

From these observations, we conclude that conventional EM fixing methods have limited capability to scale performance. Below sevenyear MTTF, these methods are only useful for fixing EM at the cost of increased area, and cannot create sufficient EM slack to increase $F_{\max }$. Physical design engineers may be able to reduce the time taken for these conventional fixes by reducing MTTF.

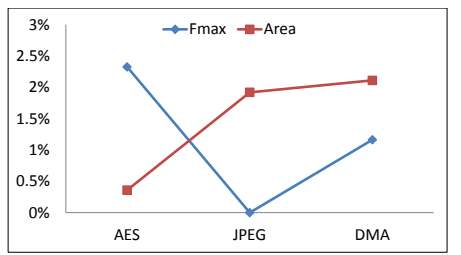

Fig. 12. Percentage increase in $F_{\max }$ and area after NDR re-route at $45 \mathrm{~nm}$.

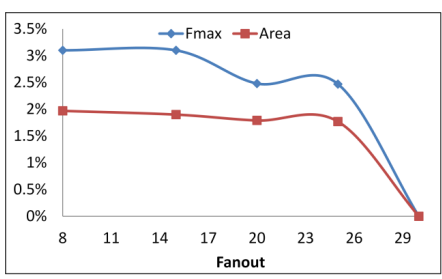

(a)

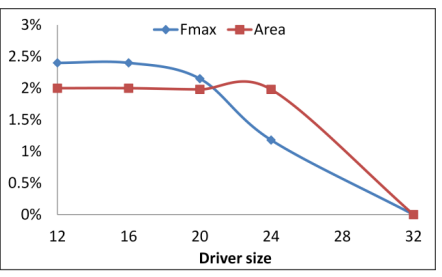

(b)
Fig. 13. Percentage increase in $F_{\max }$ and area of AES by varying (a) fanout and (b) driver size.

\section{CONClusions And Future Work}

Electromigration is a significant reliability concern, and limits performance and current density scaling in leading-edge technology nodes. In this work, we study the potential impacts of improved EM-awareness in designs, through two basic experiments that seek understanding of MTTF versus $F_{\max }$ tradeoffs at fixed resource budgets, and MTTF versus area and power tradeoffs at fixed performance requirement. We use a fully automated flow based on commercial SP\&R tools to find the maximum $F_{\max }$ for a given block implementation under EM reliability, peak temperature and area bounds; using this flow, we extract and validate trends using three designs across two technology nodes.

A key observation from our first experiment is that the available performance scaling from MTTF reduction is dependent on EM slack, which is affected by circuit characteristics such as length of critical paths, timing constraints and cell mixes in critical paths. We also show the dominating constraints (area, temperature or EM) that can limit $F_{\max }$ scaling at different lifetimes for different types of circuits. Our results further shed light on the available range of performance scaling, e.g., by reducing lifetime from 10 years to one year it is possible to increase $F_{\max }$ by $80 \%$ given the same amount of EM slack. Results of our second experiment indicate that area and power will decrease when MTTF is reduced only in (parts of) designs where timing constraints are loose. Additional studies shed light on the performance and area impact of conventional EM fix methods at a reduced MTTF requirement of seven years (with EM lifetimes below seven years, such methods offer no performance benefit in the technologies that we have studied). Specifically, performance can be increased by less than $5 \%$ with $2 \%$ increase in area when using NDRs to fix EM violations, down to a lifetime bound of seven years. Such results may give physical designers insight into when to trade off turnaround time spent on conventional EM fixes for increase in performance.

Our ongoing work studies the potential performance, area and power impacts when EM reliability requirements are relaxed for designs that have multiple operating modes - in particular, frequency overdrive modes that strongly stress interconnect reliability. Our end goal is to understand the combined impact of EM and other back end of line reliability mechanisms (time-dependent dielectric breakdown, stressinduced voiding, etc.) on interconnect lifetime and resilient design mechanisms at the architecture and system levels. ACKNOWLEDGMENTS

Research supported in part by funding from NSF, SRC, MARCO/DARPA, Qualcomm and Samsung.

\section{REFERENCES}

[1] K. Banerjee and A. Mehrotra, "Coupled analysis of electromigration reliability and performance in ULSI signal nets" Proc. ICCAD, 2001, pp. 158-164.

[2] J. R. Black, "Electromigration failure modes in aluminium metallization for semiconductor devices", IEEE Letters 57(9) (1969), pp. 1587-1594.

[3] J. J. Clement, "Electromigration modeling for integrated circuit interconnect reliability analysis", IEEE TDMR 33(1) (2001), pp. 33-42.

[4] A. Dasgupta and R. Karri, "Electromigration reliability enhancement via bus activity distribution", Proc. DAC, 1996, pp. 353-356.

[5] R. L. de Orio, H. Ceric and S. Selberherr, "Physically based models for electromigration: From Black's equation to modern TCAD models", Microelectronics Reliability 50 (2010), pp. 775-789.

[6] W. Hunter, "The implications of self-consistent current density design guidelines comprehending electromigration and Joule heating for interconnect technology evolution", Proc. IEDM, 1995, pp. 483-486.

[7] G. Jerke and J. Lienig, "Hierarchical current-density verification in arbitrarily shaped metallization patterns of analog circuits", IEEE TCAD 23(1) (2004), pp. 80-90.

[8] J. Lienig and G. Jerke, "Current-driven wire planning for electromigration avoidance in analog circuits", Proc. ASPDAC, 2003, pp. 783-788.

[9] B.-K. Liew, N. W. Cheung and C. Hu, "Electromigration interconnect lifetime under AC and pulse DC stress", Proc. IRPS, 1989, pp. 215-219.

[10] J. R. Lloyd, "Black's law revisited: Nucleation and growth in electromigration failure", Microelectronics Reliability 47 (2007), pp. 1468-1472.

[11] Z. Lu, J. Lach, M. Stan and K. Skadron, "Banking chip lifetime: Opportunities and implementation", Proc. 1st Workshop on HPCRI, 2005.

[12] Z. Lu, W. Huang, M. R. Stan, K. Skadron and J. Lach, "Interconnect reliability prediction for reliability-aware systems", IEEE TVLSI 15(2), 2007, pp. 159-172.

[13] C. A. Papameletis, "Development of design methodologies and CAD tools for system-level evaluation of interconnect reliability issues in SoC designs", Diploma Thesis, National Technical University of Athens, 2010.

[14] B. F. Romanescu and D. J. Sorin, "Core cannibalization architecture: Improving lifetime chip performance for multicore processors in the presence of hard faults", Proc. PACT, 2008, pp. 43-51.

[15] M. Shatzkes and J. R. Lloyd, "A model for conductor failure considering diffusion concurrently with electromigration resulting in a current exponent of 2", Journal of Applied Physics 59(11) (1986), pp. 3890-3893.

[16] K. Skadron, M. R. Stan, W. Huang, S. Veluswamy, K. Sankaranarayanan and D. Tarjan, "Temperature-aware microarchitecture", Proc. ISCA, 2003, pp. 2-13.

[17] J. Srinivasan, S. V. Adve, P. Bose and J. A. Rivers, "The case for lifetime reliabilityaware microprocessors", Proc. ISCA, 2004, pp. 276-287.

[18] K.-C. Wu, M.-C. Lee, D. Marculescu and S.-C. Chang, "Mitigating lifetime underestimation: A system-level approach considering temperature variations and correlations between failure times", Proc. DATE, 2012, pp. 1269-1274.

[19] W. Wu, J. S. Yuan, S. H. Kang and A. S. Oates, "Electromigration subjected to Joule heating under pulsed DC stress", Solid-State Electronics 45 (2001), pp. 2051-2056.

[20] "Clock routing rules." http://www.cadence.com/Community/blogs/di/archive/ 2011/05/10/five-minute-tutorial-setting-up-clock-routing-rules.aspx

[21] "Signal and design integrity whitepaper." http://http://w2.cadence.com/whitepapers

[22] "Virtuoso Ultrasim full-chip simulator netlist-based electromigration voltage drop (EMIR) flow." http://http://w2.cadence.com/whitepapers

[23] ' $28 \mathrm{~nm}$ IC design: The devil is in the details.' http://www.cadence.com/Community/ blogs/di/archive/2011/03/14/28-nm-ic-design-the-devil-is-in-the-details.aspx

[24] "Eliminating routing congestion issues with logic synthesis." http://www.cadence.com/rl/resources/white_papers/routing_congestion_wp.pdf

[25] "Cadence SOC Encounter user guide." http://www.cadence.com/products/di/ first_encounter/pages/default.aspx

[26] "Signal electro-migration analysis and fixing research in IC Compiler." http://www.synopsys.com.cn/information/snug/2011/ signal-electro-migration-analysis-and-fixing-research-in-ic-compiler-2

[27] "Advanced design closure techniques using IC Compiler and Zroute on high performance designs at $32 \mathrm{~nm}$ and below." https://www.synopsys.com/news/pubs/snug/austin2011/pb2_han_paper.pdf

[28] "Understand and avoid electromigration (EM) \& IR-drop in custom IP blocks." http://www.synopsys.com/Tools/Verification/CapsuleModule/CustomSim-RA-wp.pdf

[29] "Synopsys Design Compiler user guide." http://www.synopsys.com/Tools/ Implementation/RTLSynthesis/DCUltra/pages/default.aspx

[30] "SDC user's guide." http://www.actel.com/documents/SDC_AN.pdf

[31] "ITRS 2011 edition reports." http://www.itrs.net/Links/2011ITRS/Home2011.htm

[32] "LEF DEF reference." http://www.si2.org/openeda.si2.org/projects/lefdef

[33] "OpenCores." http://www.opencores.org

[34] "Americans replace their cell phones every 2 years, Finns - every six, a study claims." http://www.phonearena.com/news/Americans-replace-their-cellphones-every-2-years-Finns-every-six-a-study-claims_id20255

[35] "When should you buy a new laptop?" http://www.pcworld.com/article/219826/ when should you buy a new laptop.html

[36] "How often should you plan to replace your servers?" http://www.xennix.com/howoften-should-you-plan-to-replace-your-servers 